\title{
A volumetric full-color display realized by frequency upconversion of a transparent composite incorporating dispersed nonlinear optical crystals
}

\author{
Bin Zhu ${ }^{1,5}$, Bin $\mathrm{Qian}^{2,3}$, Yin Liu ${ }^{1,6}$, Cheng $\mathrm{Xu}^{1}$, Chang Liu ${ }^{1,2}$, Qiuqun Chen ${ }^{4}$, Jiajia Zhou ${ }^{2,3}$, Xiaofeng Liu ${ }^{1,2}$ \\ and Jianrong Qiu ${ }^{2,3}$
}

Popular three-dimensional (3D) TV or film media primarily relies on misleading our visual system by presenting our two eyes with spatially offset two-dimensional (2D) images. In comparison, volumetric displays generate moving objects in three physical dimensions with unlimited viewing angles. In a static volumetric display, voxels instead of pixels are usually addressed by luminescence, scattering or deflection. Although various prototype volumetric display technologies have been developed, the generation of full-color moving objects remains a challenge. Herein, we demonstrate the generation of voxels by frequency upconversion based on second-harmonic generation (SHG) in nonlinear optical crystals that are dispersed in solid-state composite materials that serve as a transparent solid display. Notably, voxels that radiate all colors with near-monochromatic color purity can be created by pumping at different near-infrared wavelengths and thus enable a simple solution to realize a full-color display. A computer-controlled scanner allows the generation of moving 3D objects that are viewable from any direction in a prototype device at a $25 \times 25 \times 25 \mathrm{~mm}^{3}$ scale, and larger displays that are based on the colloidal dispersion of SHG crystals are envisioned. Our methodology may have important implications for the application of the transparent crystal-in-glass composites in both 3D and 2D display technologies.

NPG Asia Materials (2017) 9, e394; doi:10.1038/am.2017.89; published online 30 June 2017

\section{INTRODUCTION}

Three-dimensional (3D) TVs and films are quickly gaining popularity in recent years across the globe. In fact, when watching a 3D movie using headgear or glasses, our eyes are seeing spatially offset two-dimensional (2D) images that are recorded from different angles and that are combined by our visual system and perceived as $3 \mathrm{D} .{ }^{1-3}$ These devices are known as stereoscopic displays that bear only partial 3D elements, including psychological depth cues of motion parallax and binocular disparity depth cues. Additionally, these technologies are often plagued by limitations, such as small view zoom, eye fatigue and necessity of wearing headgear or glasses. True 3D displays should allow for the visualization of real moving objects in three physical dimensions with complete physiological depth cues, such as accommodation, convergence and motion parallax.,4 These conditions are provided by holographic displays that are based on diffractive optics that can reconstruct the light field of the 3D objects from the holograms that are either recorded in a medium, that is, photorefractive polymer, or generated by a computer. However, the display of moving objects of a reasonable size at normal video rates remains a tremendous challenge. 5,6

In comparison, the voxels in volumetric displays are addressed by luminescence, scattering or light deflection in three physical dimensions. ${ }^{3}$ There are generally two different types of techniques: swept volume and static volume. In the swept volume display, the $3 \mathrm{D}$ image that is observed by our eyes is the result of the fusion of a series of rapidly oscillating or rotating slices due to the human persistence of vision. ${ }^{7,8}$ The static volume display has been regarded as the most 'direct' 3D display technology for a visual representation of 3D objects that employs lasers or highly collimated light to generate a visible voxel using rapid scanning of an open or closed volume that is filled with a solid, a gas (fog or smoke) or a liquid (a colloid). ${ }^{9-11}$ Infrared lasers that are invisible to the human eye have been used to generate a visible emission spot by upconversion using rare earth (RE) ion-doped transparent solids, such as glass, $, 9,10,12$ or by generating a glowing plasma in a gas or liquid phase. ${ }^{11,13,14}$ Because of the relative long lifetimes of the $4 \mathrm{f}$ levels, the frequency upconversion of RE ions provides improved

\footnotetext{
${ }^{1}$ School of Materials Science and Engineering, Zhejiang University, Hangzhou, China; ${ }^{2}$ State Key Lab of Modern Optical Instrumentation, Hangzhou, China; ${ }^{3}$ College of Optical Science and Engineering, Zhejiang University, Hangzhou, China and ${ }^{4}$ State Key Laboratory of Luminescent Materials and Devices and Institute of Optical Communication Materials, South China University of Technology, Guangzhou, China

${ }^{5}$ Current address: Corning Incorporated, Corning, NY 14831, USA.

${ }^{6}$ Current address: Department of Materials Science and Engineering, University of California, Berkeley, Berkeley, CA 94720, USA.

Correspondence: Professor X Liu, School of Materials Science and Engineering, Zhejiang University, Hangzhou 310027, China.

E-mail: xfliu@zju.edu.cn

or Professor J Quu, State Key Lab of Modern Optical Instrumentation and College of Optical Science and Engineering, Zhejiang University, Hangzhou 310027, China.

E-mail: qjr@zju.edu.cn

Received 28 November 2016; revised 17 March 2017; accepted 15 April 2017
} 
efficiency with high variation in spectral character. However, the limited availability of energy levels in RE ions precludes the emission of the three primary colors in a single block of glass under near-infrared pumping that is indispensable for the development of a full-color display. In principle, the use of a layered device architecture with diode array pumping (proposed by Downing et al. ${ }^{9}$ ) can overcome this limitation; however, such complicated devices have never been realized using existing technologies.

In contrast to the upconversion emission by RE ions that emit at certain discrete energies, second-harmonic generation (SHG) in nonlinear optical (NLO) crystals removes these limitations and, in principle, can generate emission at any wavelength with high color purity (monochromatic light) as long as a suitable fundamental beam is present. SHG can be produced in diverse molecules and crystalline materials. ${ }^{15}$ Among these materials, non-centrosymmetric nonlinear crystals generate frequency upconversion with high efficiency through the parametric process. In this work, we demonstrated the generation of a full spectrum of visible light from a transparent medium that incorporated dispersed NLO crystals, that is, a transparent glass-ceramic (GC) material that can serve as the volumetric medium for a full-color 3D display. Using a pulsed laser as the excitation source, luminescent voxels with high color purity were generated by SHG around the laser focal area, and 3D objects were displayed by computer-controlled scanning of the focus area within the active medium. Displays that are developed based on this technology support all the important psychological and physiological depth cues of a 3D display, and the methodology described herein has the potential for large-scale demonstration.

\section{MATERIALS AND METHODS}

Synthesis of a transparent GC that incorporates SHG crystals In this study, we chose a GC material that is based on fresnoite-type $\mathrm{Ba}_{2} \mathrm{TiSi}_{2} \mathrm{O}_{8}$ (BTS) crystals for reasons that are discussed in the next section. First, we synthesized the parent glass with a composition of $43 \mathrm{BaO}-18 \mathrm{TiO}_{2}-$ $36 \mathrm{SiO}_{2}-3 \mathrm{Al}_{2} \mathrm{O}_{3}$ (mol \%) using a melting-quenching process from $4 \mathrm{~N}$ purity powders. The raw materials were thoroughly mixed and then melted in air in a platinum crucible at $1450{ }^{\circ} \mathrm{C}$ for $2 \mathrm{~h}$. Then, the melt was quenched by pouring it into a steel mold that was preheated to $680^{\circ} \mathrm{C}$. After natural cooling to $\sim 300^{\circ} \mathrm{C}$, the as-formed glass was heated at $680{ }^{\circ} \mathrm{C}$ for over $12 \mathrm{~h}$ to remove internal stresses. The as-obtained glass was transparent and free of cracks and bubbles. The precipitation of BTS SHG crystals was performed by heat treatment in the temperature range between the glass transition $\left(T_{\mathrm{g}}, 700{ }^{\circ} \mathrm{C}\right)$ and crystallization $\left(T_{\mathrm{x}}, 810^{\circ} \mathrm{C}\right)$ for different durations. Finally, the as-synthesized transparent GC materials were cut and finely polished into plates with a size of $5 \times 10 \times 10 \mathrm{~mm}^{3}$ for the optical measurements. Large GC samples with a size of $25 \times 25 \times 25 \mathrm{~mm}^{3}$ based on the same composition were prepared by a similar process, except for the mechanical stirring that was applied during glass melting.

\section{Material characterization methods}

Before crystallization using thermal treatment, a differential scanning calorimetry curve of the parent glass was first recorded using the TA Instrument Q100 (New Castle, DE, USA) differential scanning calorimetry system to locate the glass transition and crystallization temperatures. To identify the precipitated crystals, the GCs were crushed into powders and examined using X-ray diffraction that employed a Rigaku D/MAX-RA diffractometer (Tokyo, Japan) with $\mathrm{Cu}-\mathrm{K} \alpha$ radiation. The microstructures of the GC powders were observed using the JEOL 2010F (scanning) transmission electron microscope (Tokyo, Japan) system to confirm the crystalline phase of the material. The transmission spectra were collected using the Hitachi U-4100 spectrophotometer (Tokyo, Japan), and the turbidity of the GCs was calculated after excluding the absorption loss.

\section{SHG generation and demonstration for volumetric displays}

A regeneratively amplified $800 \mathrm{~nm}$ Ti:sapphire laser, which emits $120 \mathrm{fs}, 1 \mathrm{kHz}$ mode-locked pulses, was used as the pumping source. The laser beam was focused into the interior of the samples using an optical lens with a $100 \mathrm{~mm}$ focal length along the longitudinal direction of the samples, and the SHG signal that radiated from the focal area was collected and recorded using the ZOLIX SBP300 spectrophotometer (Beijing, China). Emission spot images under $800 \mathrm{~nm}$ fs laser excitation were captured with a Nikon microscope (Eclipse 80i) (transmission mode) (Tokyo, Japan) that was equipped with a charge coupled device (CCD) camera. To demonstrate SHG in the blue $(450 \mathrm{~nm})$, green $(540 \mathrm{~nm})$ and red $(615 \mathrm{~nm})$ spectral regions, the fs laser beam at 900 , 1080 and $1230 \mathrm{~nm}$, respectively, was generated using a TOPAS-C optical parametric amplification system (Santa Clara, CA, USA). Three-dimensional images were created inside the GC using a computer-controlled galvanometer optical scanner that scanned the laser focus area at high speed inside the GC.

\section{RESULTS}

\section{Concept and material realization}

Inorganic glasses are the best choice among the transparent solids for optical and display applications because of their high stability and compositional flexibility. Additionally, through solid-state reactions in the glassy phases, various crystals with controllable sizes can be 'synthesized' and homogeneously dispersed within the glass matrix,

NIR laser @ 1230 nm, 1080 nm, 900 nm
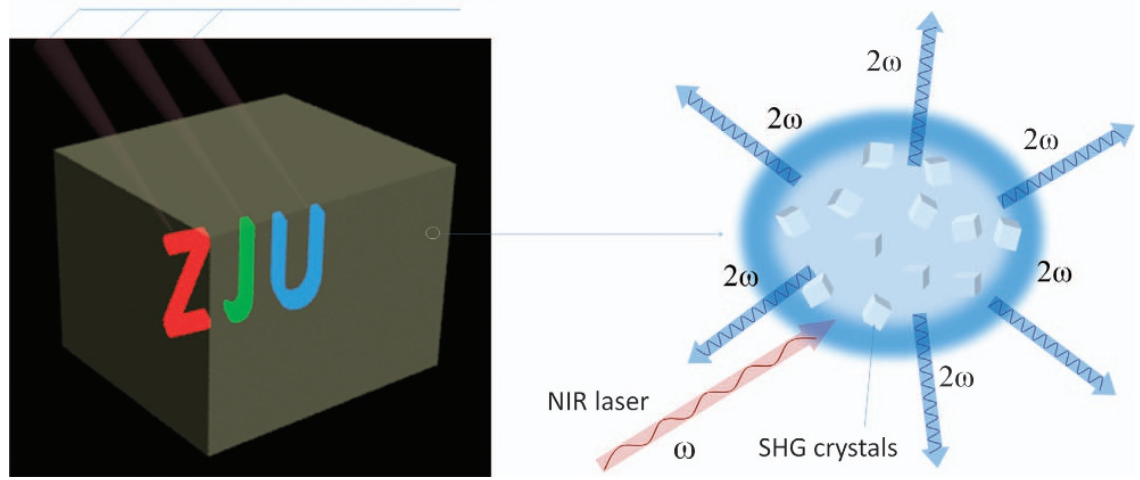

Figure 1 Schematic illustration of the working principle of the full-color volumetric display using transparent glass-ceramic (GC) as the display volume. Near-infrared (NIR) pulsed lasers were employed to generate the frequency-doubled emission that was radiated from only the focal area inside the GC. It is crucial and practically feasible that the second-harmonic generation (SHG) upconversion was confined in the focal area, such that the computer-controlled rapid scanning of the focal volume can create three-dimensional (3D) images. The SHG crystals are 'invisible' as they are dispersed in an index-matched glassy matrix. 
a
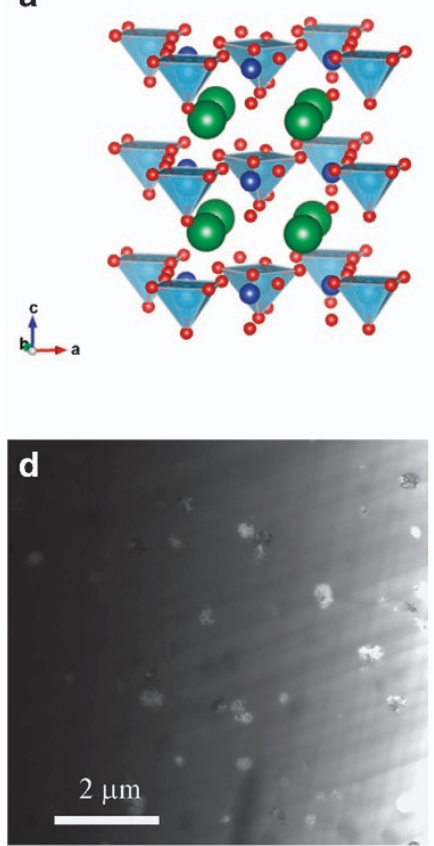

b

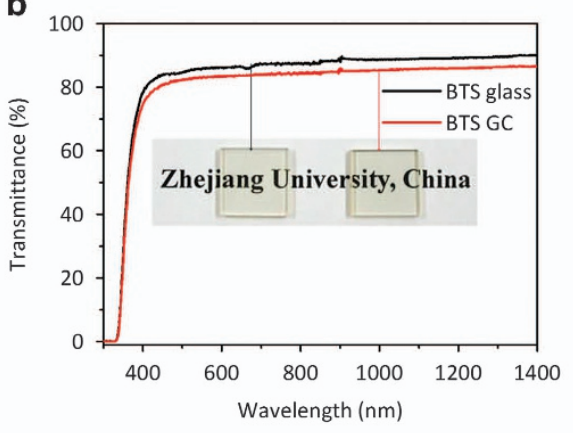

C

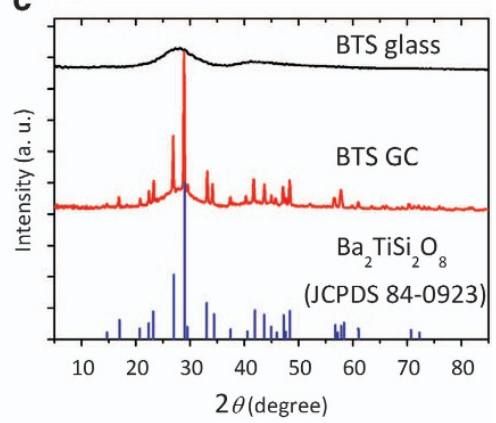

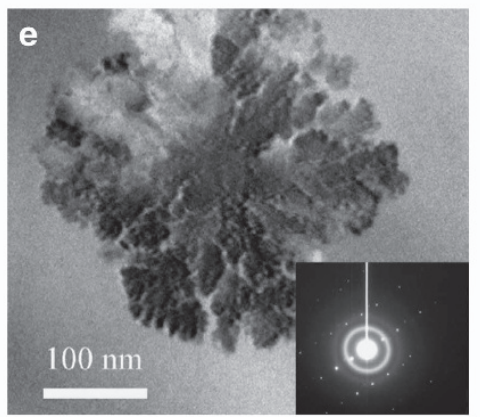

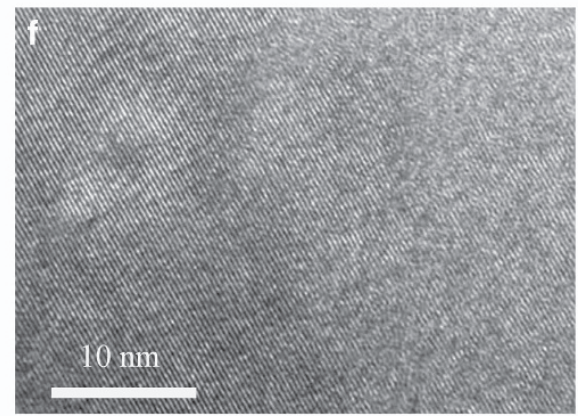

Figure 2 Characterizations of the $\mathrm{Ba}_{2} \mathrm{TiSi}_{2} \mathrm{O}_{8}$ (BTS) glass-ceramic (GC). (a) Crystal structural of the fresnoite-type BTS, where the second-harmonic generation (SHG) originated from the $\mathrm{TiO}_{5}$ pyramidal polyhedrons that were aligned along the $c$-axis. (b) Transmission spectrum of the GC and its parent glass. High transparency is retained across the entire visible range with a small scattering attenuation. (c) X-ray diffraction (XRD) patterns of the glass and GC. All of the peaks were assigned to the BTS phase. (d-f) Transmission electron microscope (TEM) images (d, e) showing typical spherulitic BTS particles with a size of $\sim 200 \mathrm{~nm}$ without a preferred orientation, and high-resolution TEM (HRTEM) image (f) showing lattice fringes that correspond to BTS.

resulting in a transparent crystal-in-glass composite known as GC that demonstrates new functionalities that are not found in the parent glass. At the core of our volumetric display is the GC-containing NLO crystals that are capable of SHG. To precipitate large crystals for higher SHG while preserving high optical transparency, we limited our selection of GC to those glass systems that congruently crystallize. In these GC systems, the glass and crystalline phases share the same chemical composition and thus offer a well-matched refractive index. Therefore, the GCs can tolerate the presence of large-sized crystals without a drastic loss in transparency by scattering. The SHG crystals with random orientations that precipitated in the GCs produced isotropic SHG signals that radiated in all directions. In addition to the laborious growth process, single crystals are considered unsuitable for display applications because SHG emission is strongly anisotropic that can cause image distortion and limit the viewing zoom (of the display). The numerous randomly oriented NLO crystals that were distributed in the glass allowed the SHG emission to be seen from any direction (Figure 1). Furthermore, in contrast to emission by active ions, which is limited by the available energy levels, SHG can generate any color with high color purity. In contrast, the parabolic dependence of SHG in the pumping power and the presence of the energy threshold enabled the confinement of the SHG emission spot in the focal area (Figure 1), allowing the generation of $3 \mathrm{D}$ images by the rapid scanning of the focal point of the near-infrared laser.

After a thorough survey of possible GC systems, we selected BTS glass that can easily form GC materials with a small index mismatch (1.77 for glass and 1.76 for crystal at $632.8 \mathrm{~nm}){ }^{16,17}$ According to scattering theory, the turbidity that characterizes the light attenuation can be described as follows. ${ }^{18}$

$$
\sigma=\frac{14}{15 \pi} \varphi(1-\varphi) k^{8} R^{7}\left(\frac{\Delta n}{n}\right)^{2}
$$

where $\varphi$ is the volume fraction of the crystalline phase, $R$ is the crystal radius, $n$ is the refraction index of the crystal, $\Delta n$ is the refractive index difference between the glassy and crystal phases and $k=2 \pi / \lambda$ ( $\lambda$ is the wavelength of light). This equation provided a rough estimation of light attenuation due to scattering in GC materials. For a BTS GC that contains a volume fraction of $5 \%$ crystalline phase with an average crystal size of $50 \mathrm{~nm}$, the turbidity is $9 \times 10^{-4} \mathrm{~cm}^{-1}$ that can account for a scattering loss that was $<1 \%$ for a 10 -mm-thick GC plate. Even for a GC with a crystal size of $200 \mathrm{~nm}$ and $\varphi=0.2$, the calculated scattering attenuation was still lower than $30 \%$, maintaining the prerequisite condition for high transparency in the GC for the proposed volumetric display. More importantly, the unique crystal structure of this compound provides a high second-order optical nonlinearity compared with that of $\mathrm{LiNbO}_{3}$ that is connected with the interconnected $\mathrm{TiO}_{5}$ pyramidal units along the $c$-axis (Figure 2a).

Initially, a sample of transparent BTS GC was synthesized for structural and spectral studies using the conventional meltingquenching process combined with thermal treatment. The condition for thermal treatment was carefully selected by referring to the differential scanning calorimetry curve of this system (Supplementary Figure S1). Then, a series of control experiments were conducted under different thermal treatment conditions using the precipitated crystals with various sizes and volume fractions. Compared with the parent glass, the as-synthesized GC (a sample that was obtained under optimized thermal treatment conditions is shown in Figure 2b) was bulk-crystallized and retained its high transparency (T\% loss of $\sim 3 \%$ ). Because of increased scattering, the GC samples crystallized at higher temperatures for longer durations, and in general, showed a smaller transmittance, especially in the short wavelength region, in agreement with the scattering law (Supplementary Figure S2). The presence of BTS crystals with a fresnoite-type structure was confirmed by X-ray diffraction (Figure 2c) 
that indicates the absence of a preferred orientation that allows isotropic SHG for the BTS GC. In Figure 2d, the transmission electron microscope image shows that the spherulitic BTS particles that precipitated inside the GC material are aggregates of the smaller BTS crystals with their $c$-axis oriented along the spherulite radius. ${ }^{19}$ Clearly, these crystalline particles are well organized that can be inferred from the single-crystal-like diffraction pattern (Figure 2e) and the high-resolution transmission electron microscope image (Figure 2f). Spherulitic crystal formation is the result of competition between the preferred growth along the $c$-axis and spatial constraint of the rigid glassy phase. However, unlike micrometer-sized crystals that were previously reported in $\mathrm{LiNbO}_{3}$ - and $\mathrm{LaBGeO}_{5}$-based GCs, ${ }^{19-21}$ the size of the BTS particles $(\sim 100-300 \mathrm{~nm})$ was smaller than the coherent length of BTS crystal $(\sim 10 \mu \mathrm{m})$, making it difficult to achieve the exact phase-matching condition for all of the particles.

\section{Frequency upconversion in the BTS GC by SHG}

The frequency upconversion of the BTS GC was examined using the above-mentioned GC chip with a $5 \mathrm{~mm}$ thickness, and the fs laser $(800 \mathrm{~nm}, 1 \mathrm{kHz})$ was employed as the pumping source. By focusing the laser beam inside the GC chip, a bright spot with strong purplecolored emission was generated; however, the laser path remained invisible to the human eye. For a closer observation of the emission spot, a CCD was employed to capture the intensity distribution around the focus of the front and side (with respect to the direction of the fundamental laser beam) surfaces of the BTS GC plate (inset in Figure $3 \mathrm{a}$ and additional details in Supplementary Figure S3). Although the fundamental beam $(800 \mathrm{~nm})$ was linearly polarized, the frequency-doubled emission was obviously isotropic when observed from the front surface. Moreover, a near-isotropic intensity distribution pattern was also generated when observed from the side surface. The observation of the direction-independent intensity distribution was possibly aided by the random orientation and distribution of the BTS nanocrystals. This capability for the generation of localized, isotropic emission spot is vital for volumetric displays because it allows a nearly unlimited viewing angle for observers.

In Figure 3, the spectrum demonstrates a peak centered at $400 \mathrm{~nm}$ with a considerable bandwidth $(\sim 10 \mathrm{~nm})$ that originates from the line width of the fundamental beam at $800 \mathrm{~nm}$. Next, the power dependence of the SHG was checked under the same measurement conditions. Similar to the two-photon upconversion process, a parabolic dependence of the SHG intensity on the pumping power was observed (as shown in Figure 3a) that again verified that the observed blue emission comes from SHG rather than diffraction or photoluminescence. The slight deviation from the ideal second-order process (slope: 1.8 vs 2) can be rationalized by a scattering effect. In particular, the SHG process was distinguished from the emission process from $\mathrm{RE}$ ions by the ultrafast response time $(\sim 100 \mathrm{fs})$, as shown in Figure 3b. This response time is also crucial for display technology as it avoids ghost images and image drifting that have plagued RE-based luminescent materials because of the extended lifetime of the $4 \mathrm{f}$ levels ( $\mu$ s to ms range).

Furthermore, the dependence of SHG on the characteristics of the transparent GC was examined. A series of GC materials with high transparency were produced under different conditions to grow NLO crystals of varying volume fractions and crystal sizes, as can be inferred a

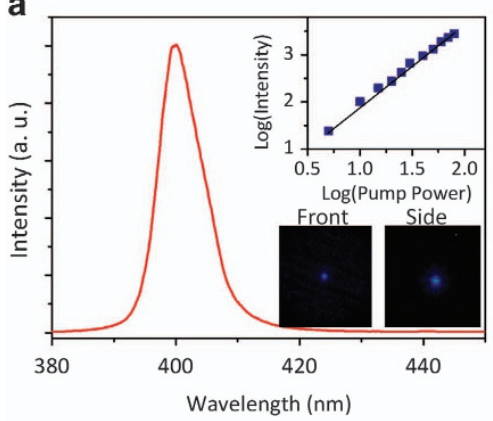

C

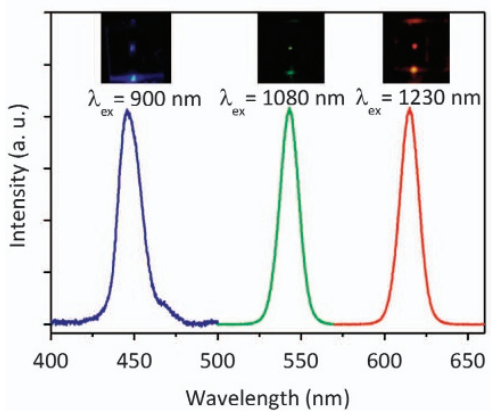

b

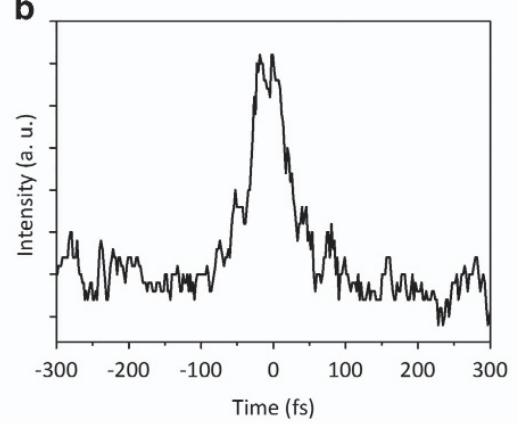

d

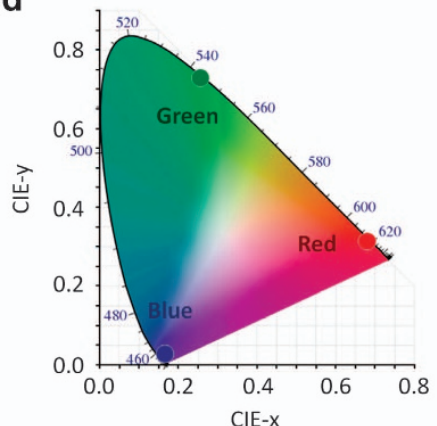

e
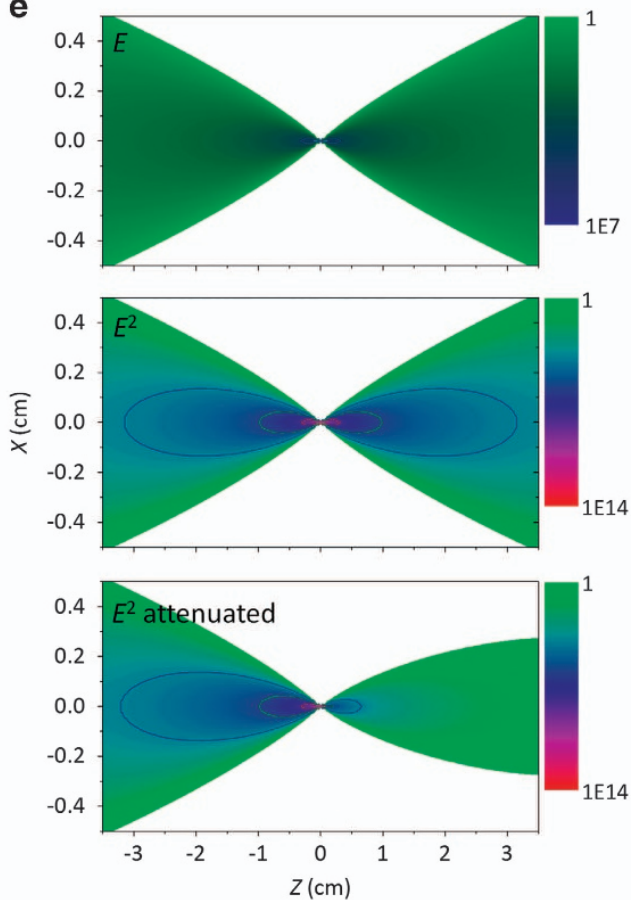

Figure 3 Second-harmonic generation (SHG) from the $\mathrm{Ba}_{2} \mathrm{TiSi}_{2} \mathrm{O}_{8}$ (BTS) glass-ceramic (GC). (a) Spectrum of the upconverted luminescence from the focal area upon irradiation using an $800 \mathrm{~nm}$ fs laser. The insets show the power dependence of the spectral intensity of the SHG signal (top) and the charge coupled device (CCD)-captured images for the emission spot from the front and side surfaces of the GC (bottom). (b) Temporal intensity profile of the SHG radiation. (c) Spectra collected from the focal area upon excitation at 900, 1080 and $1230 \mathrm{~nm}$. The inset images shows clear blue, green and red light radiated from the focal area. (d) The corresponding CIE chromaticity diagram showing the color coordinates that were calculated from the spectra shown in c. (e) Simulated electric field $E$ (upper panel) and $E^{2}$ (middle panel) intensity distribution along the light path and $E^{2}$ (bottom panel) intensity distribution in the presence of attenuation (assuming a nonlinear absorption coefficient of $0.000005 \mathrm{~cm}^{-1}$ ). The simulation was conducted by assuming a Gaussian beam profile. The red, green and blue curves represent the boundaries where the intensity is enhanced $10^{5}, 10^{7}$ and $10^{9}$ times, respectively. 
from the peak intensity of the X-ray diffraction pattern (Supplementary Figure S4). Under the same pumping condition, the increase in the SHG intensities closely matched the growth in the intensity of the diffraction peaks that again confirmed the SHG origin of the emission (Supplementary Figure S5). In addition, the growth of the SHG intensity with an increase in crystal size and volume fraction also agrees well with the theory for SHG in particulate samples. 22,23

Using SHG, the visible light at arbitrary wavelengths can be generated from the BTS GC provided that a suitable fundamental beam is present. In addition to the purple-colored emission, we also demonstrated blue, green and red emissions using an optical parametric amplification system. Fundamental beams at 900, 1080 and $1230 \mathrm{~nm}$ produced emission at 450, 540 and $615 \mathrm{~nm}$, respectively, with similar spectral widths (Figure 3c). Significantly, the emissions were nearly monochromatic and therefore offered the highest color purity, as can be seen from the color coordinates (extracted from the spectra in Figure 3c) presented in Figure 3d. Therefore, the combination of the three primary colors with near-monochromatic color purity enabled the generation of a full-color spectrum that is necessary for full-color displays.

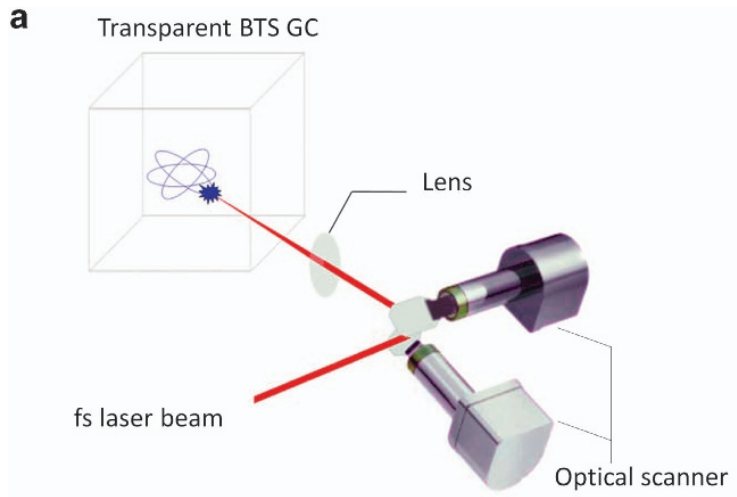

b

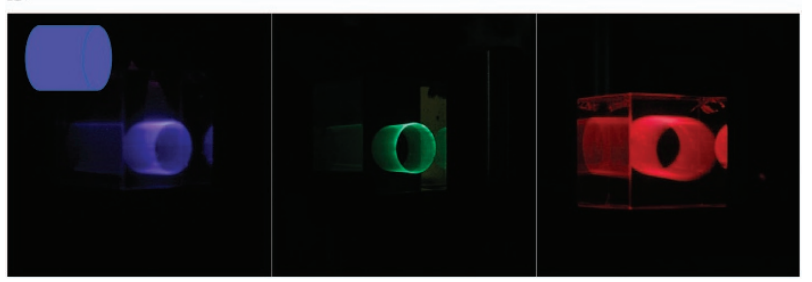

Figure 4 Creation of three-dimensional (3D) objects inside the transparent glass-ceramic (GC). (a) Schematic of the computer-controlled scanning system that employs two galvanometer mirrors and an optical lens to control light deflection and focus. (b) Blue (left), green (middle) and red 3D (right) objects were created by fast moving the laser focus using a computercontrolled scanner. The inset represents the object image that was generated by the computer.
Another feature is that the SHG emission is confined within the focal area that is indispensable for displaying high-resolution images. The resolution of a volumetric display is determined by the voxel size. Because frequency upconversion is confined within the focal area, the voxel dimension is connected with the focus size and is expressed as $d=4 \lambda f /(\pi D)$, where $\lambda$ is the wavelength, $f$ is the focal length of the lens and $D$ is the diameter of the fundamental beam. As defined by this equation, the theoretical voxel size can reach $12.7 \mathrm{~mm}$ at $1000 \mathrm{~nm}$ (for $f=10 \mathrm{~cm}, D=1 \mathrm{~cm})$ that allows for a resolution of $\sim 780 \times 780 \times 780$ for a volumetric display with a size of $1 \times 1 \times 1 \mathrm{~cm}^{3}$.

Better insight into the voxel size and shape addressed inside the transparent GC by SHG can be obtained by simulation. Figure 3e (upper panel) shows the simulated electric field intensity distribution along the light path, where the scattering attenuation is not considered. If the SHG signal scales with $I^{2}$ ( $I$ : intensity of the fundamental beam) and continuously radiates along the light path, then the intensity distribution of the SHG signal can be calculated, as shown in Figure $3 \mathrm{e}$ (middle panel). The results indicate that the area where $I^{2}$ is enhanced by $10^{5}$ has a length of only $0.98 \mathrm{~cm}$ (the length is further shortened to $0.3 \mathrm{~cm}$ for a $10^{7}$ enhancement), implying the strong localized upconversion by SHG. In view of practical applications, we further simulated the intensity distribution of $I^{2}$ in the presence of a small attenuation (for example, scattering or nonlinear absorption). The bottom panel in Figure $3 \mathrm{e}$ shows that the intensity was sharply reduced after passing the focal area that led to a further reduction in the voxel size. Furthermore, it is anticipated that the use of a lens with a shorter focal length and a large-sized beam can produce shorter voxels. In practice, the confinement of the SHG signal within the focal area is possible because SHG only emerges when the electric field intensity is greater than the threshold value. The effect of scattering, which is not included in the simulation, can lead to the attenuation in both the fundamental beam and the SHG signal; however, the small scattering (because of a close index-match) in the developed GC does not affect the observed SHG emission that is radiated from the focal area.

\section{Proof-of-concept demonstration}

As a proof-of-concept demonstration of the volumetric display, we prepared a BTS GC with dimensions of $2.5 \times 2.5 \times 2.5 \mathrm{~cm}^{3}$ (Supplementary Figure S6) that was highly transparent in the visible and near-infrared regions except for a slight yellow coloration because of the presence of $\mathrm{Ti}^{3+}$. Moreover, we used a computer-controlled mechanical scanner to construct moving $3 \mathrm{D}$ objects by controlling the movement of the focus area of the fs laser (Figure 4a). The direction of the fundamental laser beam was controlled by two galvanometer mirrors to illuminate a specific $2 \mathrm{D}$ point $(x, y)$. The depth position $(z)$ of the voxels was specified by changing the distance between the BTS GC sample and galvanometer mirrors. Besides the galvanometer mirrors, the movement of the focus can also be controlled by different means, such as a spatial light modulator, that was recently reported. ${ }^{24}$ In Figure 4b, blue-, green- and red-colored cylindrical surfaces were

Table 1 Calculated operating parameters for the hypothetical solid and liquid volumetric displays based on frequency upconversion

\begin{tabular}{lccccc}
\hline Display type & Size $(m)$ & Crystal size $(\mathrm{nm}) /$ fraction & Transmittance $(\%)^{\mathrm{a}}$ & Resolution & Minimal emission energy $(\mathrm{mW})^{b}$ \\
\hline Solid-1 & $0.02 \times 0.02 \times 0.02$ & $20 / 0.05$ & $\sim 100$ & $1560^{3}$ & 8 \\
Solid-2 & $0.1 \times 0.1 \times 0.1$ & $50 / 0.2$ & 96 & $7800^{3}$ & $10^{3}$ \\
Liquid & $1 \times 1 \times 1$ & $50 / 0.01$ & 97 & $78000^{3}$ & $10^{6}$ \\
\hline
\end{tabular}

aFor all the calculations the index difference between the second-harmonic generation (SHG) crystal and the matrix is assumed to be 0.01 at all wavelengths. The transmittance here is at 550 nm. bMinimal emission energy is calculated by assuming that luminescence intensity for the volumetric display reaches the minimal value of $1 \mu \mathrm{W} \mathrm{mm}^{-3}$. 
generated using pumping wavelengths at 900, 1080 and $1230 \mathrm{~nm}$, respectively. The scanning was performed at high frequencies (over $30 \mathrm{~Hz}$ ) to refresh the image at a rate that is appropriate for image fusion. Additionally, a moving object demonstration based on this display was also possible; however, it was limited by the scanner hardware and software capabilities. The images were recorded in darkness, but they can also be observed by the naked eye under ambient room lighting conditions at the same pumping power. A slight image drift was observed that was caused by frequency upconversion along the light path before and after the focus. This effect can be partially overcome using an optical lens with a large size and a short focal length (resulting in a large divergence angle, as discussed above). Moreover, the image drift can be mitigated using a laser system with a smaller peak power that can eliminate self-focusing and filamentation that is associated with the nonlinear optical effect. ${ }^{25-27}$

\section{DISCUSSION}

One important issue, which concerns the reliability of our strategy for the volumetric display, is the visibility of the SHG signal that radiates from the focal area that has randomly oriented nanocrystals dispersed in glass. It is generally accepted that SHG in such a system increases in intensity with the size and volume fraction of the nonlinear crystals in the glass. The total SHG intensity, which is generated by BTS crystals that are dispersed in the glass, is a complex function of the crystal size, volume fraction, nonlinear coefficient and polarization. The total SHG intensity can be simply expressed as follows: ${ }^{22}$

$$
I_{2, \text { tot }}=A f T_{2} T_{1}^{2} I_{0}^{2} L
$$

where $A$ is a constant that is determined by the size and optical constants (that is, SHG coefficients and refractive index of BTS crystals), $f$ is the volume fraction of the crystalline phase, $T_{1}$ and $T_{2}$ are the fundamental beam and SHG signal transmissions and $L$ is the thickness of the sample. Therefore, the observed efficiency of upconversion emission by SHG (defined as $I_{2 \text {,tot }} / I_{0}$ ) linearly scales with the power density of the fundamental beam and sample characteristics, such as transmittance, thickness and crystallization fraction. This implies that for large-sized displays that are based on GC materials, the scattering loss must be minimized because it exponentially scales with the sample thickness. Assuming that the upconversion emission by SHG is confined in the focal volume $\left(1 \mathrm{~mm}^{3}\right)$ that forms the voxel, a SHG efficiency of $0.003 \%$ (pumping: $100 \mathrm{~mW}$ ) would be adequate to generate emission with power of $1 \mu \mathrm{W}$ per $1 \mathrm{~mm}^{3}$ spot (corresponding to a spot radiant intensity of $5.2 \times 10^{-6} \mathrm{~lm} \mathrm{sr}^{-1}$ ) in a $2 \times 2 \times 2 \mathrm{~cm}^{3}$ BTS GC sample that is the minimum value that is required to observe green light $(540 \mathrm{~nm})$ in a dimly lit room. ${ }^{10}$ Table 1 lists the optical performance of several hypothetical solid and liquid 3D displays. Considering that the required laser power to drive the display scales with $L^{3}$ ( $L$ : size of the display), a large size display is unrealistic because the required laser power can be over $100 \mathrm{~W}$. However, only the surface of the object has to be shown for a 3D display, and therefore the laser power scales with $L^{2}$. In this regard, the required laser power can be dramatically reduced and becomes almost realizable.

In contrast, note that a large-sized GC with high optical homogeneity requires a laborious fabrication process and that the fabrication of a larger GC volume that is greater than $10 \mathrm{~cm}^{3}$ is technically difficult. An alternative to the transparent GC is a colloidal system that contains dispersed SHG nanocrystals that can be easily accessed using a wet-chemistry method (for example, sol-gel process). In fact, a colloidal solution that contains RE-doped GC nanocrystals has been proposed for the demonstration of a liquid volumetric display ${ }^{28}$ and can be easily scaled up to a large volume. Similar to the GC materials, the scattering attenuation of light can be minimized using an indexmatched solvent and SHG crystals of smaller size $(<20 \mathrm{~nm})$. Compared with solid-state displays, a colloidal display can be easily scaled up to a large size without compromising the optical performance (Table 1).

The final discussion addresses the potential application of transparent BTS GC materials to 2D transparent displays. Recently, transparent displays, such as the head-up display device, ${ }^{29,30}$ have entered the civilian market and can display navigational information on the windshield of vehicles. The use of a transparent GC that contains SHG crystals for this type of display offers several advantages, such as full color and unlimited viewing angles. Furthermore, unlike large volumes of GC with an excessive thickness, a large-sized GC plate can be easily fabricated using commercially viable technologies. Alternatively, the display can also be a transparent index-matched polymer film that contains SHG crystals that can be coated onto any planar or curved surface to enable similar 2D transparent displays.

\section{CONCLUSIONS}

In summary, we have shown that a transparent composite that contains randomly orientated NLO crystals demonstrated nearly isotropic frequency upconversion based on SHG that is localized in the laser focus area. Therefore, the SHG emission (at wavelength $\lambda$ ) from the transparent GC can cover the entire visible spectrum with nearly monochromatic color purity provided that a fundamental beam with the right wavelength $(2 \lambda)$ is present. Using computer-controlled scanners, a cubic volume of the GC enabled a proof-of-concept demonstration of a volumetric 3D display that is viewable from any angle without the need for glasses. The method disclosed here may open a new pathway for the development of both $3 \mathrm{D}$ and $2 \mathrm{D}$ transparent displays that are based on glassy materials.

\section{CONFLICT OF INTEREST}

The authors declare no conflict of interest.

\section{ACKNOWLEDGEMENTS}

This research was supported by the National Natural Science Foundation of China (grant no. 51472091, 61475047, 11504323). We thank Professor Dr E Wu of East China Normal University for assistance with the optical measurements. We also thank Professor Dr XG Liu of National University of Singapore for his valuable comments during the preparation of the manuscript.

1 Okoshi, T. Three-Dimensional Imaging Techniques (Atara Press, 2011).

2 Dodgson, N. Autostereoscopic 3D displays. Computer 38, 31-36 (2005).

3 Geng, J. Three-dimensional display technologies. Adv. Opt. Photonics 5, 456-535 (2013).

4 Holliman, N. S., Dodgson, N. A., Favalora, G. E. \& Pockett, L. Three-dimensional displays: a review and applications analysis. IEEE T. Broadcast 57, 362-371 (2011).

5 Blanche, P. A., Bablumian, A., Voorakaranam, R., Christenson, C., Lin, W., Gu, T., Flores, D., Wang, P., Hsieh, W. Y., Kathaperumal, M., Rachwal, B., Siddiqui, O., Thomas, J., Norwood, R. A., Yamamoto, M. \& Peyghambarian, N. Holographic threedimensional telepresence using large-area photorefractive polymer. Nature 468, 80-83 (2012).

6 Tay, S., Blanche, P. A., Voorakaranam, R., Tunc, A. V., Lin, W., Rokutanda, S., Gu, T., Flores, D., Wang, P., Li, G., St Hilaire, P., Thomas, J., Norwood, R. A., Yamamoto, M. \& Peyghambarian, N. An updatable holographic three-dimensional display. Nature 451, 695-698 (2008).

7 Miyazaki, D., Shiba, K., Sotsuka, K. \& Matsushita, K. Volumetric display system based on three-dimensional scanning of inclined optical image. Opt. Express 14, 12760-12769 (2006). 
8 Langhans, K., Bahr, D., Bezecny, D., Homann, D., Oltmann, K., Guill, C., Rieper, E. \& Ardey, G. FELIX 3D display: an interactive tool for volumetric imagingProc. SPIE 4660, 176-190 (2002).

9 Downing, E., Hesselink, L., Ralston, J. \& Macfarlane, R. A three-color, solid-state, three-dimensional display. Science 273, 1185-1189 (1996).

10 Lewis, J. D., Verber, C. M. \& McGhee, R. B. A true three-dimensional display. IEEE T Electron Dev. 18, 724-732 (1971).

$11 \mathrm{Kim}$, I., Korevaar, E. \& Hakakha, H. Three-dimensional volumetric display in rubidium vapor. Proc. SPIE 2650, 274-284 (1996).

12 Chen, D. Q., Wang, Y. S., Zheng, K. L., Guo, T. L., Yu, Y. L. \& Huang, P. Bright upconversion white light emission in transparent glass ceramic embedding $\mathrm{Tm}^{3+} / \mathrm{Er}^{3+} / \mathrm{Yb}^{3+}$ : beta-YF 3 nanocrystals. Appl. Phys. Lett. 91, 251903 (2007).

13 Chekhovski, A. \& Toshiyoshi, H. 3-dimensional water display. IEICE Electron. Expr. 4 430-434 (2007).

14 Koudsi, B., Refai, H. \& Sluss, J. J. Jr. Scalable upconversion medium for static volumetric display. J. Disp. Technol. 11, 266-272 (2015).

15 Shen, Y. R. The Principles of Nonlinear Optics (Wiley: New York, 1984).

16 Takahashi, Y., Kitamura, K., Benino, Y., Fujiwara, T. \& Komatsu, T. Second-order optical nonlinear and luminescent properties of $\mathrm{Ba}_{2} \mathrm{TiSi}_{2} \mathrm{O}_{8}$ nanocrystallized glass. Appl. Phys. Lett. 86, 091110 (2005).

17 Takahashi, Y., Benino, Y., Fujiwara, T. \& Komatsu, T. Large second-order optical nonlinearities of fresnoite-type crystals in transparent surface-crystallized glasses. J. Appl. Phys. 95, 3503-3508 (2004).

18 Hendy, S. Light scattering in transparent glass ceramics. Appl. Phys. Lett. 81 1171-1173 (2002).

19 Vigouroux, H., Fargin, E., Gomez, S., Le Garrec, B., Mountrichas, G., Kamitsos, E., Adamietz, F., Dussauze, M. \& Rodriguez, V. Synthesis and multiscale evaluation of $\mathrm{LiNbO}_{3}$-containing silicate glass-ceramics with efficient isotropic SHG response. Adv. Funct. Mater. 22, 3985-3993 (2012).

20 Truong, L. N., Dussauze, M., Fargin, H., Santos, L., Santos, L., Vigouroux, H. Fargues, A., Adamietz, F., Dussauze, M. \& Rodriguez, V. Isotropic octupolar second harmonic generation response in $\mathrm{LaBGeO}_{5}$ glass-ceramic with spherulitic precipitation. Appl. Phys. Lett. 106, 161901 (2015).

21 Jain, H. transparent ferroelectric glass-ceramics. Ferroelectrics 306, 111-127 (2004).

22 Aramburu, I., Ortega, J., Folcia, C. L. \& Etxebarria, J. Second-harmonic generation in dry powders: a simple experimental method to determine nonlinear efficiencies under strong light scattering. Appl. Phys. Lett. 104, 071107 (2014).
23 Aramburu, I., Ortega, J., Folcia, C. L. \& Etxebarria, J. Second harmonic generation by micropowders: A revision of the Kurtz-Perry method and its practical application. Appl. Phys. B 116, 211-233 (2014).

24 Kumagai, K., Suzuki, D., Hasegawa, S. \& Hayasaki, Y. Volumetric display with holographic parallel optical access and multilayer fluorescent screen. Opt. Lett. 40, 3356-3359 (2015).

25 Tan, D. Z., Sharafudeen, K. N., Yue, Y. Z. \& Qiu, J. R. Femtosecond laser induced phenomena in transparent solid materials: Fundamentals and applications. Prog. Mater. Sci. 76, 154-228 (2016).

26 Kosareva, O. G., Kandidov, V. P., Brodeur, A. \& Chin, S. L. From filamentation in condensed media to filamentation in gases. J. Nonlinear Opt. Phys. Mater. 6, 485-494 (1997).

27 Brodeur, A. \& Chin, S. L. Ultrafast white-light continuum generation and self-focusing in transparent condensed media. J. Opt. Soc. Am. B 16, 637-650 (1999).

28 Liu, X. F., Dong, G. P., Qiao, Y. B. \& Qiu, J. R. Transparent colloid containing upconverting nanocrystals: an alternative medium for three-dimensional volumetric display. Appl. Opt. 47, 6416-6421 (2008).

29 Newman, R. L. Head-Up Displays: Designing the Way Ahead (Ashgate Publishing: Surrey, 1995).

30 Hsu, C. W., Zhen, B., Qiu, W. J., Shapira, O., DeLacy, B. G., Joannopoulos, J. D. \& Soljačić, M. Transparent displays enabled by resonant nanoparticle scattering. Nat. Commun. 5, 3152 (2014).

(i) This work is licensed under a Creative Commons Attribution 4.0 International License. The images or other third party material in this article are included in the article's Creative Commons license, unless indicated otherwise in the credit line; if the material is not included under the Creative Commons license, users will need to obtain permission from the license holder to reproduce the material. To view a copy of this license, visit http:// creativecommons.org/licenses/by/4.0/

(C) The Author(s) 2017

Supplementary Information accompanies the paper on the NPG Asia Materials website (http://www.nature.com/am) 